\title{
Trail Making Test Part A and Brain Perfusion Imaging in Mild Alzheimer's Disease
}

\author{
Aki Shindo ${ }^{a}$ Seishi Terada ${ }^{a}$ Shuhei Sato ${ }^{b}$ Chikako Ikeda $^{a}$ \\ Shigeto Nagao ${ }^{a}$ Etsuko Oshima ${ }^{a}$ Osamu Yokota ${ }^{a}$ Yosuke Uchitomi ${ }^{a}$ \\ Departments of a Neuropsychiatry and ${ }^{b}$ Radiology, Okayama University Graduate School of \\ Medicine, Dentistry and Pharmaceutical Sciences, Okayama, Japan
}

Key Words

Alzheimer's disease - Cerebral blood flow - Single photon emission computed tomography ·

Trail Making Test

\section{Abstract}

Background/Aims: The Trail Making Test (TMT) has long been used to investigate deficits in cognitive processing speed and executive function in humans. However, there are few studies that elucidate the neural substrates of the TMT. The aim of the present study was to identify the regional perfusion patterns of the brain associated with performance on the TMT part $A$ (TMT-A) in patients with Alzheimer's disease (AD). Methods: Eighteen AD patients with poor performance on the TMT-A and 36 age- and sex-matched AD patients with good performance were selected. All subjects underwent brain single photon emission computed tomography. Results: No significant differences between the good and poor performance groups were found with respect to years of education and revised Addenbrooke's Cognitive Examination scores. However, higher z-scores for hypoperfusion in the bilateral superior parietal lobule were observed in the group that scored poorly on the TMT-A compared with the good performance group. Conclusion: Our results suggest that functional activity of the bilateral superior parietal lobules is closely related to performance time on the TMT-A. Thus, the performance time on the TMT-A might be a promising index of dysfunction of the superior parietal area among mild $A D$ patients. 


\section{Introduction}

Alzheimer's disease (AD) is the leading cause of late-onset dementia worldwide. Patients with AD display a multiplicity of cognitive deficits in domains such as memory, visuospatial cognition, language, and executive function [1]. Therefore, in the dementia clinic, many neuropsychological tests have been used to detect deficits in cognitive function. When evaluating the results of those tests, it is important to know the neural correlates of the tests. The Trail Making Test (TMT) is among the most commonly used neuropsychological tests in clinical practice, including memory clinics [2]. The TMT has long been used to investigate deficits in cognitive processing speed and executive function in humans [3]. However, there is relatively more ambiguity about the neural systems associated with the TMT than with many other tests of comparable clinical use [4], and there have been no studies to investigate the neural substrates of the TMT among patients with AD.

To elucidate the neural substrate of neuropsychiatric symptoms in $\mathrm{AD}$, brain single photon emission computed tomography (SPECT) is often used. Dorsolateral prefrontal hypoperfusion is involved in depression [5-7], whereas medial frontal or orbitofrontal hypoperfusion is devoted to apathy [7-9]. Delusion is closely related to decreased perfusion in the right frontal lobe [10-12], and anosognosia may be a reflection of functional impairment in the orbitofrontal or inferior frontal cortex [13-15]. The neural substrate of neuropsychological tests for patients with dementia has also been disclosed using brain SPECT data. The total Mini-Mental State Examination (MMSE) score is significantly correlated with a reduction of left hippocampal perfusion [16]. The Frontal Assessment Battery score and the categoryachieved score from the Wisconsin Card Sorting Test are closely related to functional activity of the dorsolateral prefrontal areas $[17,18]$.

In this study, we tried to clarify the relationship between TMT time and regional cerebral blood flow (rCBF) using SPECT data. The TMT consists of part A and part B (TMT-A and TMT-B). As a first step, we investigated the cerebral correlates of the TMT-A. Studies of the mutual relationships among various neuropsychological tests report that the TMT-A examines mainly visuoperceptual abilities and graphomotor speed $[3,19]$. However, in another study, participants with frontal brain damage performed significantly more poorly than those with nonfrontal brain damage on the TMT-A but not on the TMT-B [20]. In a study using ${ }^{18}$ F-fluorodeoxyglucose positron emission tomography (FDG-PET), the focal brain metabolic rate did not predict the performance of schizophrenia patients $(n=42)$ on the TMT-A [21]. In this study, we aimed to compare the rCBF of AD patients with good and poor performances on the TMT-A, and to investigate the neural substrate involved in the performance of AD patients on the TMT-A. Because mutual relationships among various neuropsychological tests suggest that visuospatial ability is very important for the performance of the TMT-A, and visual attention deficits in AD have been reported to correlate with superior parietal lobe hypoperfusion $[1,22]$, we predicted a relationship between the TMT-A performance time and $\mathrm{rCBF}$ in the parietal region among AD patients.

\section{Methods}

\section{The Trail Making Test}

The TMT-A and TMT-B were administered by clinical psychologists who were blind to the clinical diagnosis and Clinical Dementia Rating (CDR) score. The tests were conducted according to the standard administration procedure described by Spreen and Strauss [23].

Briefly, the TMT-A requires the patient to link in ascending order a series of 25 numbers $(1,2,3 \ldots)$ randomly distributed in space [24]. Subjects are instructed to start their trial at the 
circle marked 'Begin' and continue linking numbers until they reach the endpoint (circle marked 'End'). In the Japanese version of the TMT-B, the test is modified by changing letters of the Roman alphabet (A, B, C...) to Kana (Japanese phonograms; ' 'a', 'i', ' 'u' ...) [25]. The TMT-B is similar to the TMT-A, although instead of just linking numbers, the patient must alternately switch between a set of numbers (1-13) and a set of Kana letters (' $a$ ' through 'shi'), again linking them in ascending order (1/a/2/i...). During the tests, the examiner corrects each error immediately. At the same time, the subject is also asked to connect the array of circles as fast as possible without lifting the pencil [24]. The time limits for performing the TMT-A and TMT-B are 300 and $600 \mathrm{~s}$, respectively.

The detailed normative TMT data stratified by age and educational level have been reported elsewhere [26]. In this study, patients with a TMT-A time greater than or equal to the mean +2 SD for the normal elderly were included in the poor performance group on the TMT-A, while patients with a TMT-A time less than or equal to the mean + 1 SD for the normal elderly were included in the good performance group on the TMT-A.

\section{Subjects}

Eighteen AD patients with dementia severity of 0.5 (suspicious) or 1 (mild) based on the CDR [27] and whose time on the TMT-A was over the mean + 2 SD were selected from 124 consecutive $\mathrm{AD}$ patients who were fully examined at the Memory Clinic of Okayama University Hospital between April 2004 and March 2010. In addition, 36 AD patients from the Memory Clinic with good performance on the TMT-A, matched for age, sex, education, and revised Addenbrooke's Cognitive Examination (ACE-R) scores [28, 29], were also selected.

They all (1) underwent general physical and neurological examinations and extensive laboratory testing, including thyroid function tests, serum vitamin $\mathrm{B}_{12}$, and syphilis serology; (2) took the MMSE [30] and ACE-R within 1 week of scanning; (3) underwent brain SPECT as well as head MRI; (4) were diagnosed with probable AD according to the criteria formulated by the NINCDS-ADRDA [31]; (5) had a dementia severity of 0.5 or 1 on the CDR [27], and (6) they or their nearest relatives gave written informed consent.

The exclusion criteria were (1) complications by other neurological diseases or illnesses; (2) a history of mental illness or substance abuse prior to the onset of dementia; (3) any evidence of focal brain lesions on head MRI; (4) treatment with cholinesterase inhibitors, antipsychotics, antidepressants, or anxiolytic drugs, and (5) left-handedness or ambidexterity.

Information was also gathered from caregivers familiar with the patient's daily behavior. To serve as an informant, the caregiver must have been living with the patient. The profile of each subject (age, sex, years of education, and years of disease duration) was recorded. The Neuropsychiatric Inventory (NPI) [32], Physical Self-Maintenance Scale (PSMS), and Lawton Instrumental Activities of Daily Living (IADL) scale [33] were scored based on the information from caregivers.

\section{Other Instruments}

The ACE-R was developed to provide a brief test sensitive to early-stage dementia and is capable of differentiating between dementia subtypes including AD, frontotemporal dementia, progressive supranuclear palsy, and other parkinsonian syndromes [28]. The ACE-R includes the MMSE but extends it to encompass important areas not covered by the MMSE, such as frontal-executive function and visuospatial skills. For this study, we used the Japanese version of the ACE-R described by Yoshida et al. [29]. The reliability of the Japanese version of the ACE-R is excellent, and its validity is, to some extent, established [29].

Neuropsychiatric symptoms were evaluated by the NPI. The NPI is a valid and reliable instrument for measuring behavior in dementia [32, 34]. It is a caregiver-based tool that assesses 10 common behaviors in dementia. The PSMS and IADL are validated scales for the 
Shindo et al.: Trail Making Test Part A and Brain Perfusion Imaging in Mild Alzheimer's Disease

assessment of activities of daily living (ADL) [33]. The PSMS is a 6-item scale that rates selfcare ability in toileting, feeding, dressing, personal hygiene and grooming, locomotion (physical ambulation), and bathing. The IADL scale assesses a patient's ability to perform 8 complex daily tasks: ability to use the telephone, shopping, food preparation, household tasks, laundry, mode of transportation, responsibility for medications, and ability to manage finances.

\section{Ethics}

The study protocol was approved by the Committee of Okayama University Hospital on Human Research. After giving a complete description of the study to the subjects and their relatives, written informed consent was obtained.

\section{Brain Perfusion SPECT}

All subjects were examined by brain perfusion SPECT. Patients were examined in a comfortable supine position with their eyes closed in quiet surroundings. First, the passage from the heart to the brain was monitored after intravenous administration of ${ }^{99 \mathrm{~m} T c-e t h y l}$ cysteinate dimer (ECD, 600 MBq; Daiichi Radioisotope Laboratories Ltd., Tokyo, Japan). Ten minutes after the angiography, SPECT images were obtained using a triple-head, rotating gamma camera interfaced to a minicomputer (GCA9300A/DI; Toshiba, Tokyo, Japan) equipped with a fan beam, low-energy, high-resolution collimator. Sixty projection images over a $360^{\circ}$ angle in a $128 \times 128$ matrix were acquired. All images were reconstructed using ramp-filtered back projection and then three-dimensionally smoothed with a Butterworth filter (order 8 , cutoff 0.12 cycles $/ \mathrm{cm}$ ). The reconstructed images were corrected for gamma ray attenuation using the Chang method $(\mu=0.09)$.

\section{Data Analysis}

Spatial reprocessing and statistical analysis of images were performed on a voxel-byvoxel basis using Statistical Parametric Mapping 2 (SPM2, Wellcome Department of Imaging Neuroscience, London, UK) running on MATLAB (The MathWorks Inc., Natick, Mass., USA). All SPECT images of each subject were normalized to the standard brain of the Montreal Neurological Institute, and spatial normalization was performed with 12-parameter affine and nonlinear transformations [35]. The voxel sizes of the reslice option were $2 \times 2 \times 2 \mathrm{~mm}$. The nonlinear parameter was set at $25-\mathrm{mm}$ cutoff basis functions and 16 iterations. All normalized SPECT images were then smoothed with an isotropic gaussian kernel filter (12 $\mathrm{mm}$ full width at half-maximum). To examine the images for specific regions showing differences in perfusion, two-sample $t$ tests were performed. Global normalization was performed by proportional scaling with the mean voxel value. Masking was applied using the threshold method ( 0.8 times the global value). The analysis used a threshold of $\mathrm{p}<0.01$ at the voxel level, and results were considered significant at $\mathrm{p}<0.05$ (uncorrected) at the cluster level.

\section{Statistical Analysis}

Statistical analysis was performed using the SPSS 14.0J software program (SPSS Inc., Chicago, Ill., USA). The two groups were compared using independent sample t tests. A value of $\mathrm{p}<0.05$ was accepted as significant.

\section{Results}

Clinical Characteristics and Neuropsychological Tests

No significant differences were found between the good and poor performance groups on the TMT-A with respect to age $(\mathrm{p}=0.456)$ or years of education $(\mathrm{p}=0.638$; table 1$)$. 
Table 1. Clinical characteristics and neuropsychological tests

\begin{tabular}{lcccc}
\hline & Bad score & Good score & $\mathrm{t}$ & $\mathrm{p}$ \\
\hline Total & 18 & 36 & & \\
Males/females & $7 / 11$ & $10 / 26$ & & 0.206 \\
Age, years & $72.7 \pm 7.9$ & $75.3 \pm 6.7$ & -1.281 & 0.638 \\
Education, years & $11.5 \pm 3.3$ & $11.1 \pm 2.6$ & 0.473 & 0.968 \\
ACE-R score & $69.2 \pm 12.8$ & $69.1 \pm 7.4$ & 0.040 & 0.945 \\
MMSE score & $23.2 \pm 3.3$ & $23.2 \pm 2.5$ & -0.069 & 0.000 \\
TMT-A time, s & $99 \pm 23$ & $50 \pm 15$ & 9.605 & 0.002 \\
TMT-B time, s & $406 \pm 230$ & $239 \pm 144$ & 3.281 & 0.019 \\
TMT B-A time, s & $308 \pm 222$ & $189 \pm 138$ & 2.426 & 0.290 \\
TMT B/A ratio & $4.1 \pm 2.2$ & $4.8 \pm 2.3$ & -1.068 & \\
\hline
\end{tabular}

Values are $\mathrm{n}$ or means $\pm \mathrm{SD}$. Bad score $=\mathrm{AD}$ patients with poor performance on the TMT-A. Good score $=$ AD patients with good performance on the TMT-A.

Table 2. ADL and IADL

\begin{tabular}{|c|c|c|c|c|}
\hline & Bad score & Good score & $\mathrm{t}$ & $\mathrm{p}$ \\
\hline \multicolumn{5}{|l|}{ NPI } \\
\hline Delusion & $0.5 \pm 1.6$ & $0.4 \pm 1.0$ & 0.457 & 0.650 \\
\hline Hallucination & $0.3 \pm 0.8$ & $0.1 \pm 0.5$ & 0.911 & 0.367 \\
\hline Agitation/aggression & $0.3 \pm 1.0$ & $0.4 \pm 1.3$ & -0.419 & 0.677 \\
\hline Depression & $0.7 \pm 1.0$ & $0.4 \pm 0.9$ & 1.078 & 0.287 \\
\hline Anxiety & $0.2 \pm 0.4$ & $0.1 \pm 0.5$ & 0.497 & 0.621 \\
\hline Euphoria & $0 \pm 0$ & $0 \pm 0$ & 0.000 & 1.000 \\
\hline Apathy & $1.1 \pm 1.4$ & $1.1 \pm 1.5$ & 0.156 & 0.877 \\
\hline Disinhibition & $0.1 \pm 0.3$ & $0.2 \pm 0.7$ & -0.446 & 0.658 \\
\hline Irritability & $0.4 \pm 0.9$ & $0.9 \pm 1.8$ & -0.915 & 0.365 \\
\hline Aberrant motor behavior & $0.5 \pm 1.2$ & $0.2 \pm 1.1$ & 0.941 & 0.352 \\
\hline \multicolumn{5}{|l|}{ PSMS } \\
\hline Toileting & $0.9 \pm 0.3$ & $1.0 \pm 0.0$ & -2.052 & 0.045 \\
\hline Feeding & $0.9 \pm 0.2$ & $1.0 \pm 0.0$ & -1.408 & 0.165 \\
\hline Dressing & $0.8 \pm 0.4$ & $1.0 \pm 0.2$ & -1.825 & 0.074 \\
\hline Grooming & $0.9 \pm 0.3$ & $1.0 \pm 0.2$ & -1.226 & 0.226 \\
\hline Ambulation & $0.8 \pm 0.4$ & $1.0 \pm 0.2$ & -2.360 & 0.022 \\
\hline Bathing & $0.9 \pm 0.4$ & $1.0 \pm 0.0$ & -0.797 & 0.429 \\
\hline \multicolumn{5}{|l|}{ IADL } \\
\hline Ability to use telephone & $0.9 \pm 0.2$ & $1.0 \pm 0.0$ & -1.408 & 0.165 \\
\hline Shopping & $0.8 \pm 0.4$ & $0.9 \pm 0.3$ & -1.030 & 0.308 \\
\hline Food preparation & $0.7 \pm 0.5$ & $0.8 \pm 0.4$ & -0.292 & 0.772 \\
\hline Housekeeping & $1.0 \pm 0.0$ & $1.0 \pm 0.0$ & 0.000 & 1.000 \\
\hline Laundry & $0.9 \pm 0.4$ & $1.0 \pm 0.0$ & -2.018 & 0.052 \\
\hline Mode of transportation & $1.0 \pm 0.0$ & $1.0 \pm 0.0$ & 0.000 & 1.000 \\
\hline Responsibility for own medications & $0.6 \pm 0.5$ & $0.7 \pm 0.5$ & -0.752 & 0.456 \\
\hline Ability to handle finances & $0.9 \pm 0.3$ & $1.0 \pm 0.0$ & -2.052 & 0.045 \\
\hline
\end{tabular}

Values are means $\pm \mathrm{SD}$. Bad score $=\mathrm{AD}$ patients with a low score on the TMT-A. Good score $=\mathrm{AD}$ patients with a high score on the TMT-A. 
Table 3. Significant regional uptake differences between AD patients with high and low scores on the TMT-A

\begin{tabular}{lllrll}
\hline Direction of difference & $\begin{array}{l}\text { Number } \\
\text { of voxels }\end{array}$ & Peak z-scores & \multicolumn{2}{c}{ Coordinates (MNI) } \\
\cline { 4 - 6 } & & $\mathrm{x}$ & $\mathrm{y}$ & $\mathrm{z}$ \\
\hline Decreased uptake in & 553 & 3.44 & -10 & -70 & 70 \\
AD patients with low & & 3.16 & 0 & -76 & 62 \\
scores on the TMT-A & & 3.04 & -4 & -86 & 48 \\
\hline
\end{tabular}

MNI = Montreal Neurological Institute
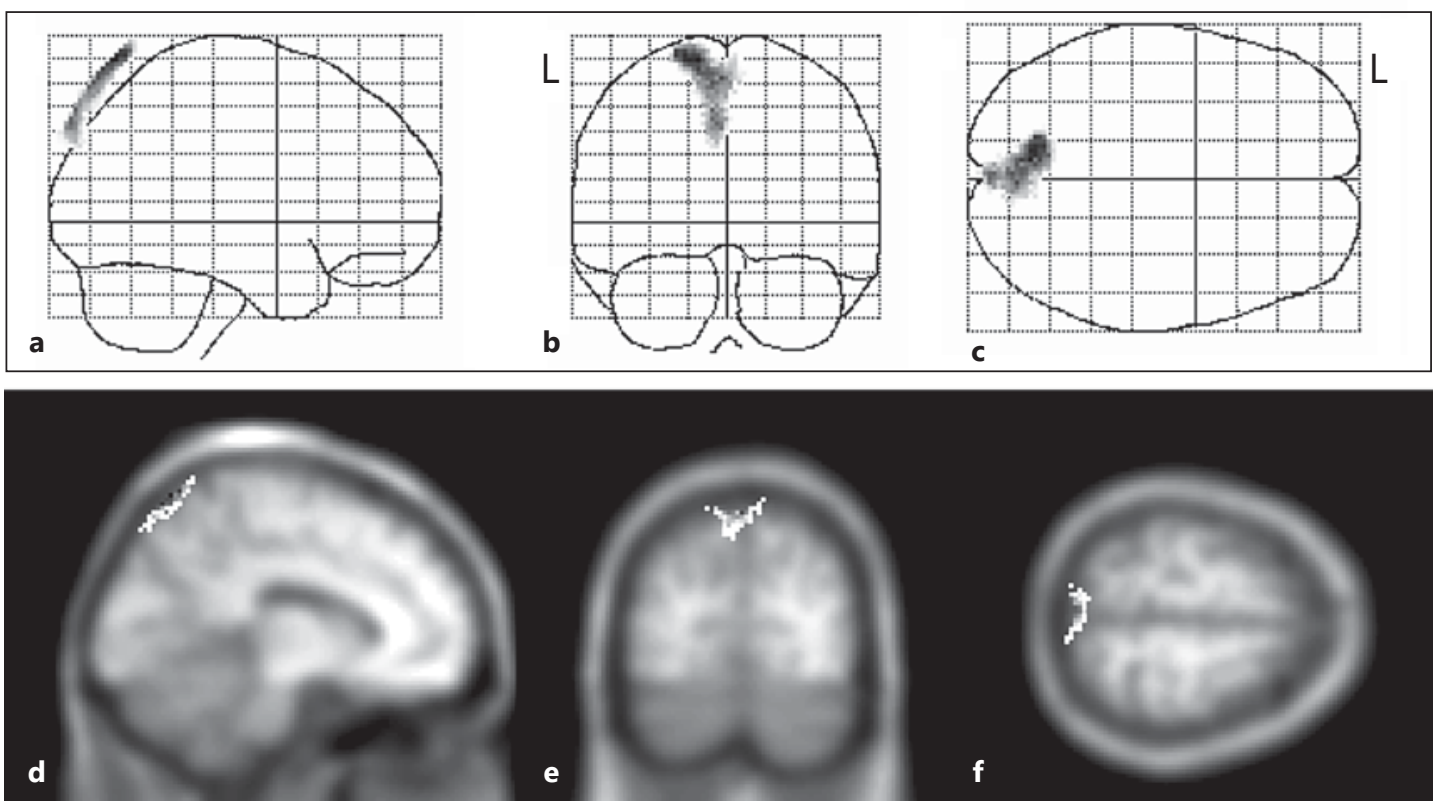

Fig. 1. a-f SPM (z) of rCBF decrease in AD patients with poor performance on the TMT-A compared with AD patients with good performance. Three-way glass view of the area of significant hypoperfusion (a-c), superimposed on head MRI T1-weighted images: $x=-10(\mathbf{d}), y=-75(\mathbf{e})$, and $z=65$ (f). L = Left.

With the exception of the TMT-A, TMT-B, and TMT B-A [(TMT-B time) - (TMT-A time)] times (TMT-A, $p<0.001$; TMT-B, $p<0.013$; TMT B-A, $p<0.019$ ), no significant differences were found between the good and poor performance groups on the ACE-R ( $p=0.968)$, MMSE $(\mathrm{p}=0.945)$, TMT B/A ratio [(TMT-B time)/(TMT-A time)] $(\mathrm{p}=0.290)$, or NPI subscale scores (table 1,2). Comparison of the PSMS and IADL scale revealed that AD patients with poor performance on the TMT-A had lower scores than those with good performance on the TMT-A in the subscale scores of toileting, physical ambulation, and ability to handle finances (table 2).

\section{Regional Cerebral Blood Flow}

A group comparison between the SPM results of the good and poor performance groups was performed. Specific voxels with a significantly lower perfusion in the poor performance group than in the good performance group are shown in figure 1 and table 3. 
Figure 1 shows the z-score for each voxel in this cluster superimposed onto a threedimensional glass brain view. A significant cluster of voxels in the left superior parietal lobule slightly extending to the right superior parietal lobule can also be seen in figure 1 . Table 2 shows the probability results of the Statistical Parametric Mapping analysis and the location of peak z-scores in terms of the Montreal Neurological Institute coordinates.

\section{Discussion}

\section{Superior Parietal Lobule and the TMT}

We predicted hypoperfusion in the parietal region among AD patients with poor performance on the TMT-A, and this study revealed that $\mathrm{rCBF}$ in the bilateral superior parietal lobule of AD patients with poor performance on the TMT-A was lower than that of AD patients with good performance on the TMT-A. Therefore, the results are in accordance with our hypotheses.

Visual attention and visuospatial processing involve the superior parietal lobule [36, 37], and both are impaired in AD [38-42]. As stated above, the TMT-A requires mainly visuoperceptual abilities and graphomotor speed $[3,19]$. Therefore, it is not surprising that performance on the TMT-A among AD patients is significantly correlated with $\mathrm{rCBF}$ in the superior parietal lobule. However, to our knowledge, this is the first study to show that rCBF in the superior parietal lobule of AD patients with poor performance on the TMT-A is significantly lower than that of $\mathrm{AD}$ patients with good performance.

TMT and $A D L$

Visual attention and visuospatial processing are reported to greatly affect patients' ADL $[42,43]$. In this study, AD patients with poor performance on the TMT-A showed lower scores than those with good performance on the TMT-A in the subscale scores of toileting, physical ambulation, and ability to handle finances among PSMS and IADL. Our results suggest that performance on the TMT-A is closely related to several ADL as well as rCBF in the superior parietal lobule, independent of ACE-R and MMSE scores.

\section{Superior Parietal Lobule and $A D$}

At the very early stage of $\mathrm{AD}$, regional cortical atrophy is mainly restricted to the medial temporal structures as well as the posterior cingulate gyrus [44-46]. With the progression of the disease, the atrophic areas extend to the parietal and frontal lobes with the advancement of the disease $[47,48]$. Significant decreases of rCBF were detected in the bilateral parietal, angular gyrus, pericallosal, thalamus, right temporal, and left hippocampal regions in mild AD [49].

We found a decrease in $\mathrm{CCBF}$ in the superior parietal lobule among mild AD patients with poor performance on the TMT-A compared to those with good performance. Our results suggest that some AD patients suffer from parietal neuropsychological deficits and parietal hypoperfusion in the early stage of the disease.

\section{Limitations of This Study}

The TMT is a popular neuropsychological test and is often used to detect executive dysfunction [3]. In those cases, the TMT B-A time or TMT B/A ratio is calculated as a score reflecting executive function $[20,50]$. However, in this study we focused on the TMT-A. We selected two groups of patients based on their TMT-A scores. As a result, the AD patients with poor performance on the TMT-A also showed lower scores on the TMT-B time and TMT B-A time but not on the TMT B/A ratio. Therefore, it cannot be denied that the TMT-B time and 
TMT B-A time are also significantly related to $\mathrm{rCBF}$ in the superior parietal lobule. Further study is needed to clarify the significance of the TMT B-A time or TMT B/A ratio by comparing rCBF between two groups with significantly different scores on the TMT B-A time or TMT B/A ratio but without significantly different scores on the TMT-A time.

The results of this study should be interpreted with some caution. First, the relatively small sample size, comprising only 18 AD patients with poor performance on the TMT-A and 36 AD patients with good performance on the TMT-A, may limit the sensitivity to actual group differences and associations between neuropsychological functions and regional brain hypoperfusion. Second, the low spatial resolution of SPECT scans may limit detection of alterations in small regions, thus increasing the risk of false negatives and interference from surrounding regions [51].

\section{Acknowledgements}

We thank Ms. Horiuchi, Ms. Imai, Ms. Yabe, and Ms. Tsuchiyama for their skillful assistance in this study. This work was supported by a grant from the Japanese Ministry of Education, Culture, Sports, Science and Technology (24591714), and the Zikei Institute of Psychiatry.

\section{References}

1 Vasquez BP, Buck BH, Black SE, Leibovitch FS, Lobaugh NJ, Caldwell CB, Behrmann M: Visual attention deficits in Alzheimer's disease: relationship to HMPAO SPECT cortical hypoperfusion. Neuropsychologia 2011;49: 1741-1750.

-2 Ashendorf L, Jefferson AL, O'Connor MK, Chaisson C, Green RC, Stern RA: Trail Making Test errors in normal aging, mild cognitive impairment, and dementia. Arch Clin Neuropsychol 2008;23:129-137.

-3 Sánchez-Cubillo I, Periáñez JA, Adrover-Roig D, Rodríguez-Sánchez JM, Ríos-Lago M, Tirapu J, Barceló F: Construct validity of the Trail Making Test: role of task-switching, working memory, inhibition/interference control, and visuomotor abilities. J Int Neuropsychol Soc 2009;15:438-450.

4 Allen MD, Owens TE, Fong AK, Richards DR: A functional neuroimaging analysis of the Trail Making Test-B: implications for clinical application. Behav Neurol 2011;24:159-171.

5 Levy-Cooperman N, Burhan AM, Rafi-Tari S, Kusano M, Ramirez J, Caldwell C, Black SE: Frontal lobe hypoperfusion and depressive symptoms in Alzheimer disease. J Psychiatry Neurosci 2008;33:218-226.

-6 Akiyama H, Hashimoto H, Kawabe J, Higashiyama S, Kai T, Kataoka K, Shimada A, Inoue K, Shiomi S, Kiriike N: The relationship between depressive symptoms and prefrontal hypoperfusion demonstrated by eZIS in patients with DAT. Neurosci Lett 2008;441:328-331.

7 Kang JY, Lee JS, Kang H, Lee HW, Kim YK, Jeon HJ, Chung JK, Lee MC, Cho MJ, Lee DS: Regional cerebral blood flow abnormalities associated with apathy and depression in Alzheimer disease. Alzheimer Dis Assoc Disord 2012;26:217-224.

-8 Benoit M, Clairet S, Koulibaly PM, Darcourt J, Robert PH: Brain perfusion correlates of the apathy inventory dimensions of Alzheimer's disease. Int J Geriatr Psychiatry 2004;19:864-869.

-9 Lanctôt KL, Moosa S, Herrmann N, Leibovitch FS, Rothenburg L, Cotter A, Black SE: A SPECT study of apathy in Alzheimer's disease. Dement Geriatr Cogn Disord 2007;24:65-72.

10 Staff RT, Venneri A, Gemmell HG, Shanks MF, Pestell SJ, Murray AD: HMPAO SPECT imaging of Alzheimer's disease patients with similar content-specific autobiographic delusion: comparison using statistical parametric mapping. J Nucl Med 2000;41:1451-1455.

-11 Nakano S, Yamashita F, Matsuda H, Kodama C, Yamada T: Relationship between delusions and regional cerebral blood flow in Alzheimer's disease. Dement Geriatr Cogn Disord 2006;21:16-21.

12 Ismail Z, Nguyen MQ, Fischer CE, Schweizer TA, Mulsant BH: Neuroimaging of delusions in Alzheimer's disease. Psychiatry Res 2012;202:89-95.

-13 Vogel A, Hasselbalch SG, Gade A, Ziebell M, Waldemar G: Cognitive and functional neuroimaging correlate for anosognosia in mild cognitive impairment and Alzheimer's disease. Int J Geriatr Psychiatry 2005;20:238-246.

14 Salmon E, Perani D, Herholz K, Marique P, Kalbe E, Holthoff V, Delbeuck X, Beuthien-Baumann B, Pelati O, Lespagnard S, Collette F, Garraux G: Neural correlates of anosognosia for cognitive impairment in Alzheimer's disease. Hum Brain Mapp 2006;27:588-597. 
Shindo et al.: Trail Making Test Part A and Brain Perfusion Imaging in Mild Alzheimer's Disease

15 Shibata K, Narumoto J, Kitabayashi Y, Ushijima Y, Fukui K: Correlation between anosognosia and regional cerebral blood flow in Alzheimer's disease. Neurosci Lett 2008;435:7-10.

-16 Ikeda E, Shiozaki K, Takahashi N, Togo T, Odawara T, Oka T, Inoue T, Hirayasu Y: Total Mini-Mental State Examination score and regional cerebral blood flow using $\mathrm{Z}$ score imaging and automated ROI analysis software in subjects with memory impairment. Ann Nucl Med 2008;22:539-542.

$\checkmark 17$ Takeda N, Terada S, Sato S, Honda H, Yoshida H, Kishimoto Y, Kamata G, Oshima E, Ishihara T, Kuroda S: Wisconsin card sorting test and brain perfusion imaging in early dementia. Dement Geriatr Cogn Disord 2010; 29:21-27.

18 Oshima E, Terada S, Sato S, Ikeda C, Nagao S, Takeda N, Honda H, Yokota O, Uchitomi Y: Frontal assessment battery and brain perfusion imaging in Alzheimer's disease. Int Psychogeriatr 2012;24:994-1001.

19 Misdraji EL, Gass CS: The Trail Making Test and its neurobehavioral components. J Clin Exp Neuropsychol 2010;32:159-163.

20 Demakis GJ: Frontal lobe damage and tests of executive processing: a meta-analysis of the Category Test, Stroop Test, and Trail-Making Test. J Clin Exp Neuropsychol 2004;26:441-450.

21 Horacek J, Dockery C, Kopecek M, Spaniel F, Novak T, Tislerova B, Klirova M, Palenicek T, Höschl C: Regional brain metabolism as the predictor of performance on the Trail Making Test in schizophrenia. A ${ }^{18}$ FDG PET covariation study. Neuro Endocrinol Lett 2006;27:587-594.

22 Buck BH, Black SE, Behrmann M, Caldwell C, Bronskill MJ: Spatial- and object-based attentional deficits in Alzheimer's disease. Relationship to HMPAO-SPECT measures of parietal perfusion. Brain 1997;120:12291244.

23 Spreen O, Strauss E: A Compendium of Neuropsychological Tests: Administration, Norms and Commentary, ed 2. New York, Oxford University Press, 1998.

24 Zakzanis KK, Mraz R, Graham SJ: An fMRI study of the Trail Making Test. Neuropsychologia 2005;43:18781886.

25 Hashimoto R, Meguro K, Lee E, Kasai M, Ishii H, Yamaguchi S: Effect of age and education on the Trail Making Test and determination of normative data for Japanese elderly people: the Tajiri Project. Psychiatry Clin Neurosci 2006;60:422-428.

-26 Harada H, Notoya M, Nakanishi M, Fujiwara N, Inoue K: Effects of age and years of education on neuropsychological data of Japanese healthy elderly persons (in Japanese). High Brain Funct Res 2006;26:16-24.

27 Hughes CP, Berg L, Danziger WL, Coben LA, Martin RL: A new clinical scale for the staging of dementia. Br J Psychiatry 1982;140:566-572.

-28 Mioshi E, Dawson K, Mitchell J, Arnold R, Hodges JR: The Addenbrooke's Cognitive Examination Revised (ACE-R): a brief cognitive test battery for dementia screening. Int J Geriatr Psychiatry 2006;21:1078-1085.

-29 Yoshida H, Terada S, Honda H, Kishimoto Y, Takeda N, Oshima E, Hirayama K, Yokota O, Uchitomi Y: Validation of the revised Addenbrooke's Cognitive Examination (ACE-R) for detecting mild cognitive impairment and dementia in a Japanese population. Int Psychogeriatr 2012;24:28-37.

-30 Folstein MF, Folstein SE, McHugh PR: 'Mini-Mental State'. A practical method for grading the cognitive state of patients for the clinician. J Psychiatr Res 1975;12:189-198.

31 McKhann G, Drachman D, Folstein M, Katzman R, Price D, Stadlan EM: Clinical diagnosis of Alzheimer's disease: report of the NINCDS-ADRDA Work Group under the auspices of Department of Health and Human Services Task Force on Alzheimer's Disease. Neurology 1984;34:939-944.

-32 Cummings JL, Mega M, Gray K, Rosenberg-Thompson S, Carusi DA, Gornbein J: The Neuropsychiatric Inventory: comprehensive assessment of psychopathology in dementia. Neurology 1994;44:2308-2314.

-33 Hokoishi K, Ikeda M, Maki N, Nomura M, Torikawa S, Fujimoto N, Fukuhara R, Komori K, Tanabe H: Interrater reliability of the Physical Self-Maintenance Scale and the Instrumental Activities of Daily Living Scale in a variety of health professional representatives. Aging Ment Health 2001;5:38-40.

34 Hirono N, Mori E, Ikejiri Y, Imamura T, Shimomura T, Hashimoto M, Yamashita H, Ikeda M: Japanese version of the Neuropsychiatric Inventory: a scoring system for neuropsychiatric disturbance in dementia patients (in Japanese). No To Shinkei 1997;49:266-271.

-35 Friston KJ, Ashburner J, Poline JB, Frith CD, Heather JD, Frackowiak RSJ: Spatial realignment and normalization of images. Hum Brain Mapp 1995;2:165-189.

36 Alivisatos B, Petrides M: Functional activation of the human brain during mental rotation. Neuropsychologia 1997;35:111-118.

-37 Vandenberghe R, Gitelman DR, Parrish TB, Mesulam MM: Functional specificity of superior parietal mediation of spatial shifting. Neuroimage 2001;14:661-673.

-38 Ska B, Poissant A, Joanette Y: Line orientation judgment in normal elderly and subjects with dementia of Alzheimer's type. J Clin Exp Neuropsychol 1990;12:695-702.

-39 Parasuraman R, Greenwood PM, Haxby JV, Grady CL: Visuospatial attention in dementia of the Alzheimer type. Brain 1992;115:711-733.

40 Rizzo M, Anderson SW, Dawson J, Myers R, Ball K: Visual attention impairments in Alzheimer's disease. Neurology 2000;54:1954-1959.

-41 Meguro K, Shimada M, Someya K, Horikawa A, Yamadori A: Hemispatial visual-searching impairment correlated with decreased contralateral parietal blood flow in Alzheimer's disease. Neuropsychiatry Neuropsychol Behav Neurol 2001;14:213-218. 
42 Prvulovic D, Hubl D, Sack AT, Melillo L, Maurer K, Frölich L, Lanfermann H, Zanella FE, Goebel R, Linden DE, Dierks T: Functional imaging of visuospatial processing in Alzheimer's disease. Neuroimage 2002;17:14031414.

43 Jackson GR, Owsley C: Visual dysfunction, neurodegenerative diseases, and aging. Neurol Clin 2003;21:709_ 728.

44 Karas GB, Burton EJ, Rombouts SA, van Schijndel RA, O’Brien JT, Scheltens P, McKeith IG, Williams D, Ballard C, Barkhof F: A comprehensive study of gray matter loss in patients with Alzheimer's disease using optimized voxel-based morphometry. Neuroimage 2003;18:895-907.

-45 Hirata Y, Matsuda H, Nemoto K, Ohnishi T, Hirao K, Yamashita F, Asada T, Iwabuchi S, Samejima H: Voxel-based morphometry to discriminate early Alzheimer's disease from controls. Neurosci Lett 2005;382:269-274.

46 Guo X, Wang Z, Li K, Li Z, Qi Z, Jin Z, Yao L, Chen K: Voxel-based assessment of gray and white matter volumes in Alzheimer's disease. Neurosci Lett 2010;468:146-150.

47 Good CD, Scahill RI, Fox NC, Ashburner J, Friston KJ, Chan D, Crum WR, Rossor MN, Frackowiak RS: Automatic differentiation of anatomical patterns in the human brain: validation with studies of degenerative dementias. Neuroimage 2002;17:29-46.

48 Guo XJ, Chen CS, Chen KW, Jin Z, Peng DL, Yao L: Brain development in Chinese children and adolescents: a structural MRI study. Neuroreport 2007;18:875-880.

49 Kobayashi S, Tateno M, Utsumi K, Takahashi A, Saitoh M, Morii H, Fujii K, Teraoka M: Quantitative analysis of brain perfusion SPECT in Alzheimer's disease using a fully automated regional cerebral blood flow quantification software, 3DSRT. J Neurol Sci 2008;264:27-33.

50 Fernández AL, Marcopulos BA: A comparison of normative data for the Trail Making Test from several countries: equivalence of norms and considerations for interpretation. Scand J Psychol 2008;49:239-246.

51 Takeuchi R, Yonekura Y, Matsuda H, Konishi J: Usefulness of a three-dimensional stereotaxic ROI template on anatomically standardised ${ }^{99 \mathrm{~m}}$ Tc-ECD SPET. Eur J Nucl Med Mol Imaging 2002;29:331-341. 